\title{
ARTICLE
}

\section{In vivo imaging of murine endocrine islets of Langerhans with extended-focus optical coherence microscopy}

\author{
M. Villiger $\cdot$ J. Goulley $\bullet$ M. Friedrich $\cdot$ \\ A. Grapin-Botton • P. Meda • T. Lasser • R. A. Leitgeb
}

Received: 19 December 2008 / Accepted: 8 April 2009 /Published online: 30 May 2009

(C) Springer-Verlag 2009

\begin{abstract}
Aims/hypothesis Structural and functional imaging of the islets of Langerhans and the insulin-secreting beta cells represents a significant challenge and a long-lasting objective in diabetes research. In vivo microscopy offers a valuable insight into beta cell function but has severe limitations regarding sample labelling, imaging speed and depth, and was primarily performed on isolated islets lacking native innervations and vascularisation. This article introduces extended-focus optical coherence microscopy (xfOCM) to image murine pancreatic islets in their natural environment in situ, i.e. in vivo and in a label-free condition.
\end{abstract}

Electronic supplementary material The online version of this article (doi:10.1007/s00125-009-1383-y) contains supplementary material, which is available to authorised users.

M. Villiger $(\bowtie) \cdot$ M. Friedrich $\cdot$ T. Lasser $\cdot$ R. A. Leitgeb Laboratoire d'Optique Biomédicale, Ecole Polytechnique Fédérale de Lausanne,

Station 17 ,

CH-1015 Lausanne, Switzerland

e-mail: martin.villiger@epfl.ch

J. Goulley · A. Grapin-Botton

Swiss Institute for Experimental Cancer Research/Ecole

Polytechnique Fédérale de Lausanne,

Lausanne, Switzerland

P. Meda

Department of Cell Physiology and Metabolism,

University of Geneva,

Medical School,

Geneva, Switzerland

R. A. Leitgeb

Center of Biomedical Engineering and Physics,

Medical University of Vienna,

Vienna, Austria
Methods Ex vivo measurements on excised pancreases were performed and validated by standard immunohistochemistry to investigate the structures that can be observed with xfOCM. The influence of streptozotocin on the signature of the islets was investigated in a second step. Finally, xfOCM was applied to make measurements of the murine pancreas in situ and in vivo.

Results xfOCM circumvents the fundamental physical limit that trades lateral resolution for depth of field, and achieves fast volumetric imaging with high resolution in all three dimensions. It allows label-free visualisation of pancreatic lobules, ducts, blood vessels and individual islets of Langerhans ex vivo and in vivo, and detects streptozotocin-induced islet destruction.

Conclusions/interpretation Our results demonstrate the potential value of XfOCM in high-resolution in vivo studies to assess islet structure and function in animal models of diabetes, aiming towards its use in longitudinal studies of diabetes progression and islet transplants.

Keywords Mouse $\cdot$ Imaging $\cdot$ Islets

$\begin{array}{ll}\text { Abbreviations } \\ \text { MRI } & \text { Magnetic resonance imaging } \\ \text { NA } & \text { Numerical aperture } \\ \text { OCM } & \text { Optical coherence microscopy } \\ \text { OCT } & \text { Optical coherence tomography } \\ \text { OPT } & \text { Optical projection tomography } \\ \text { PET } & \text { Positron emission tomography } \\ \text { xfOCM } & \text { Extended-focus optical coherence microscopy }\end{array}$

It is essential to be able to measure the structure and function of individual islets of Langerhans [1]. To further the understanding of the mechanisms of diabetes and to evaluate treatments of the disease, monitoring of functional 
islet variables is mandatory. Most applicable to a clinical setting are positron emission tomography (PET) and magnetic resonance imaging (MRI) [2-4]. Their medical potential is undoubted but, to date, only optical methods have provided the necessary spatial resolution to resolve individual islets [5]. Although most of these methods are likely to have little impact on clinical practice and human in vivo imaging, they are indispensable and complementary tools to provide insight into the cellular and molecular mechanisms of this disease.

The preparation of sections with immunocytochemical labelling is the standard method to visualise subcellular structures and to discriminate between the various islet cell types. This method is time consuming and only gives a two-dimensional view of the tissue slices. Optical projection tomography (OPT) [6] has been used to image the adult mouse pancreas and to retrieve the three-dimensional and undistorted structure of the tissue, which may be quantified. However, such approaches require sample fixation and immunolabelling and, hence, cannot be applied in vivo $[7,8]$.

Likewise, functional studies are performed in vitro on isolated islets $[9,10]$ and often after fluorochrome labelling, which is prone to bleaching and phototoxicity. Two-photon microscopy reduces these effects [11]. Even so, the islets are deprived of their natural tissue environment which limits the physiological relevance of such studies.

In recent work [12] islets have been transplanted into the mouse eye. Engrafted on the iris they could be repeatedly imaged using the eye as a natural body window. This minimally invasive approach allows longitudinal monitoring of individual islets and visualisation of islet vascularisation, beta cell function and death by using two-photon fluorescence microscopy in combination with appropriate fluorochromes and transgenic mouse models expressing green fluorescent protein.

Here, we report on the first three-dimensional in vivo imaging of the murine pancreas macro- and microstructure by an approach that requires no labelling and that works in situ. The islets are studied in their natural environment with their native innervation and vascularisation. The technique we have developed is extended-focus Fourier domain optical coherence microscopy (xfOCM) [13]. It is based on optical coherence tomography (OCT), an emerging biomedical imaging modality that provides cross-sectional views of the subsurface microstructure of biological tissue $[14,15]$. It employs light from a low coherence source that is back-scattered by differences in the tissue refractive index and that is combined with a strong reference signal. The detection in the Fourier, or frequency, domain spectrally separates the interference signal into its chromatic components and records the resulting pattern with a line camera. Processing of this pattern extracts the sample structure along the axial direction in parallel without axial scanning and with a high resolution of 2-3 $\mu \mathrm{m}$ [16]. Together with its high sensitivity [17] that allows imaging several hundred micrometres into the tissue, Fourier domain OCT provides an exceptional speed advantage over other optical imaging methods, making it a confirmed tool for comprehensive volumetric imaging [18, 19].

Classic optical coherence microscopy (OCM) [20, 21] uses standard focusing optics to obtain high lateral resolution. The resulting short depth of field hampers the parallel depth extraction of Fourier domain OCT and necessitates time-consuming scanning in all three dimensions, which makes in vivo measurements difficult. Our novel approach has been to circumvent this compromise between lateral resolution and depth of field by engineering an extended focus that is scanned rapidly in the lateral directions.

Our study validates this approach for the in vitro and in vivo imaging of islets of Langerhans and provides evidence for its potential value in structural and functional studies.

\section{Methods}

$x$ fOCM setup The prototype xfOCM system developed for this study provides a high, near isotropic resolution of $1.3 \mu \mathrm{m}$ in the lateral and $2 \mu \mathrm{m}$ in the axial direction, revealing structural details and fine cell arrangements over an axial range of $300 \mu \mathrm{m}$. The basic layout is displayed in Fig. 1. The linearly polarised light source (Ti:Sapphire laser, Femtolasers, Vienna, Austria; central wavelength: 780 $\mathrm{nm}$; spectral full width at half maximum: $130 \mathrm{~nm}$ ) is collimated $\left(\mathrm{L}_{\mathrm{S}}: f=8.2 \mathrm{~mm}\right.$ ) and split by the beamsplitter $\mathrm{BS}_{1}$ into sample and reference arm (Fig. 1). The sample beam passes through the axicon (apex angle $180^{\circ}-2 \beta=$ $\left.175^{\circ}\right)$ and is relayed $\left(\mathrm{L}_{\mathrm{R}}: f=100 \mathrm{~mm}\right)$ through the scanning system, which provides rapid lateral scanning. The two galvo scanners rotate the beam around a common pivot point and the lens $\mathrm{L}_{\mathrm{T}}(f=164 \mathrm{~mm})$ creates finally a thin annulus of radius $\approx f \beta(n-1)$ in the back aperture of the objective (Zeiss Neofluar, $\times 10$, numerical aperture [NA] 0.3; Carl Zeiss, Jena, Germany). The common pivot point of the scanning system, in a conjugate plane of the back aperture of the objective, is crucial to the correct scanning of the beam in the lateral directions. In the sample region, the different contributions converge in the proximity of the focal plane and interfere to create a Bessel-like illumination pattern in the radial direction, propagating nearly diffraction free over a long axial distance of nearly $400 \mu \mathrm{m}$ with a uniform lateral definition of $\sim 1.3 \mu \mathrm{m}$ (see small inlet in Fig. 1). The light back-scattered from the sample is decoupled from the illumination path by beamsplitter $\mathrm{BS}_{2}$ to prevent a double pass through the axicon. The descanned 


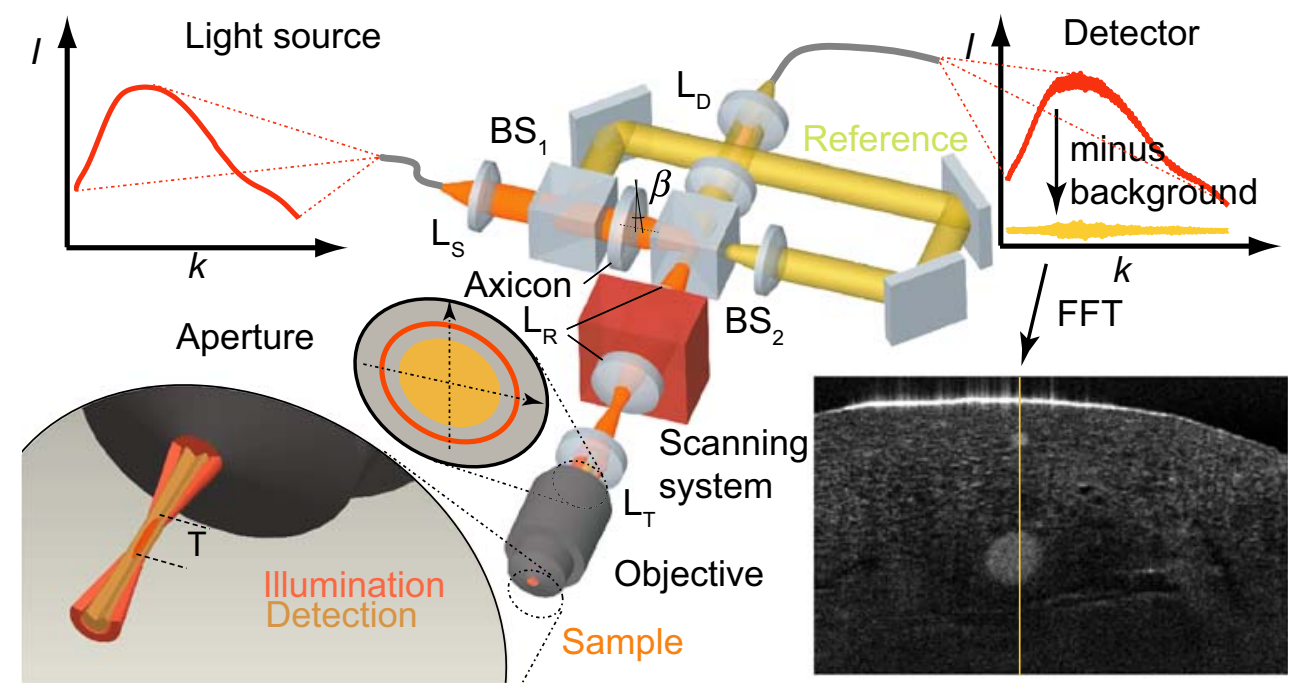

Fig. 1 Principle layout of the xfOCM setup. The incoming broadband light is collimated by lens $\mathrm{L}_{\mathrm{S}}$ and split by beamsplitter $\mathrm{BS}_{1}$ into sample and reference beam. The light in the sample arm passes through the axicon, is relayed by lenses $\mathrm{L}_{\mathrm{R}}$ through the scanning system and focused by lens $\mathrm{L}_{\mathrm{T}}$ into a thin annulus in the objective's aperture (small inlet). In the sample region, interference produces a light-needle which extends over a long axial range $\mathrm{T}$ (big inlet),

signal is combined with the reference beam and coupled into the detection fibre of NA 0.12 .

This detection scheme has a twofold impact. First, the signal is detected with high efficiency, resulting in sufficient sensitivity to image biological samples. Second, with the detection aperture smaller than the annulus diameter, light from specular reflection is suppressed, producing a dark field effect. Nevertheless, the resulting detection volume acts as apodisation on the illumination needle, producing an effective extended focus of $\sim 300 \mu \mathrm{m}$.

The signal is analysed with a spectrometer consisting of a transmission grating $(1,200$ lines $/ \mathrm{mm})$ and a linear camera with 2,048 pixels (Atmel Aviva, Stemmer Imaging, Pfäffikon, Switzerland) set to an integration time of $40 \mu \mathrm{s}$, working at a depth profile rate of $20 \mathrm{kHz}$. The illumination power on the sample was 3-4 mW. Adjacent scanning points were separated by between $0.2 \mu \mathrm{m}$ and $2.5 \mu \mathrm{m}$, varying the area of one scan between $100 \times 200 \mu \mathrm{m}$ and $2 \times 1.2 \mathrm{~mm}$.

Signal processing The signal recorded on the spectrometer can be expressed as $I(k)=\left|A_{r^{\prime}}{ }^{2}+\alpha^{2}\right| A_{s}{ }^{2}+2 \operatorname{Re}\left\{A_{r} A_{s} \times \int h(z) \mathrm{e}^{i 2 k(z-z))} \mathrm{d} z\right\}$, where $A_{r}$ and $A_{s}$ are the amplitudes of the electromagnetic field in the reference and sample arms, respectively, $h(z)$ represents the local reflectivity of the sample structure as a function of $z, k=2 \pi / \lambda$ is the wave number, and $\alpha^{2}=\left|\int h(z) e^{i 2 k(z-z 0)} d z\right|^{2}$ is the total reflectivity of the sample. To reconstruct the sample structure, we compute the Fourier transformation of the signal after subtracting the average spectral background and performing an scanning the sample in the lateral directions. The backscattered light is recombined with the light from the reference arm at beamsplitter $\mathrm{BS}_{2}$ and coupled into the detection fibre by lens $\mathrm{L}_{\mathrm{D}}$. The detected interference pattern is corrected by the reference spectrum and fast Fourier transformed (FFT) from the wave-number k-space to the axial coordinate of the sample structure

interpolation to a linear $\mathrm{k}$-space. The signal processing was performed on a $2.13 \mathrm{GHz}$ dual core Intel Xeon based personal computer with 4 GB DDRII SDRAM. The Microsoft Windows XP-based application software was developed in $\mathrm{C}++$ and made use of the Trolltech QT v4.3 framework (Trolltech, Oslo, Norway; www.trolltech.com/, accessed 4 May 2007), the GNU Scientific Library v1.8 (Free Software Foundation, Boston, MA, USA; www.gnu. org/software/gsl/, accessed 18 May 2007) and the Fastest Fourier Transform in the West (FFTW) v3.1.2 library (Massachusetts Institute of Technology, Boston, MA, USA; www.fftw.org/, accessed 5 June 2007).

All figures display the logarithm of the squared signals, and are rescaled and adjusted to optimise contrast and brightness. The $z$-axes of the tomograms were rescaled by an estimated sample index of $n=1.33$ in order to represent geometrical distances rather than optical path lengths. Apart from the in vivo images, for which an image registration was performed, no post processing was applied. Figures $2 \mathrm{~d}$ and $3 \mathrm{a}$ were produced using Voxx, a freeware threedimensional rendering program (www.nephrology.iupui. edu/imaging/voxx, accessed 4 November 2008). Electronic supplementary material (ESM) videos 3 and 4 were rendered with Imaris (Bitplane, Zürich, Switzerland).

Semi-automatic islet detection In order to perform statistical analysis, the tomograms were screened for islet-like structures. Determination of the islet volume and position was achieved by manually defining a region of interest and automatic extraction of the precise islet shape by filtering 

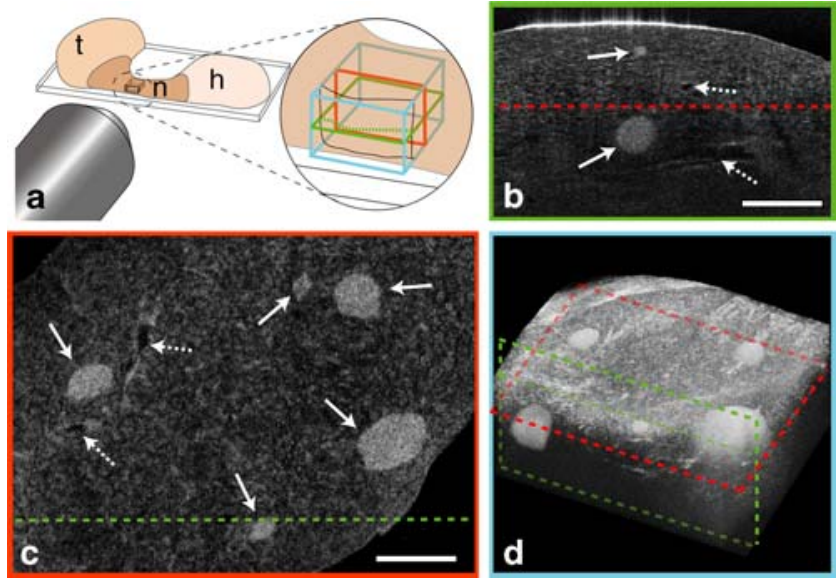

Fig. 2 Scanning principle of xfOCM. a Scanning along one lateral direction creates a tomogram along the depth and the scan axis (green rectangle). Acquiring many such tomograms by scanning along the second lateral direction provides a three-dimensional volume (blue cube). En face images can be generated by selecting one depth (red rectangle). The pancreas consists of the head $(h)$ and the tail $(t)$, connected by the neck (n). b Lateral scan of a fixed sample, measured in the neck region depicting a $1 \mathrm{~mm} \times 0.65 \mathrm{~mm}$ field (scale bar, $250 \mu \mathrm{m}$ ). Two small islets are present (arrows), along with vessel structures (dashed arrows). The bright line at the top of the tomogram indicates the sample surface that strongly reflects the incident light. c En face image at a depth of $220 \mu \mathrm{m}$ showing five islets (arrows) and vessels (dashed arrows) (scale bar, $250 \mu \mathrm{m}$ ). d The volume data can be represented with a three-dimensional rendering, giving a three dimensional impression $(1.5 \times 1 \times 0.65 \mathrm{~mm})$. See also ESM videos 1 and 2 for the complete volume data and a three-dimensional rendering

and thresholding (see ESM 1). The volumetric islet mask obtained was used to estimate the mean of the islet signal. Likewise, the mean of the signal from the exocrine tissue surrounding the islet was extracted. The difference of these two values gives a measure of the contrast of an islet. In a further step, the signal variation within the islet core was analysed to evaluate homogeneity, expressed as the lateral gradient of the mean signal in function of depth within the islet, going from the outer surface to its inner core.

Preparation of dissected pancreas All animal studies were approved by the Cantonal Veterinary Office (Vaud, Switzerland) and were conducted in accordance with Swiss animal protection law.

Pancreases $(n=13)$ from 3-month-old ICR female mice (Harlan Laboratories, Itingen, Switzerland) kept in normal housing and with food ad libidum (2018 Teklad Global $18 \%$ Protein Rodent Diet, Harlan Laboratories) were dissected. The samples were fixed for $45 \mathrm{~min}$ in a $4 \%$ (vol./vol.) paraformaldehyde solution in PBS at room temperature, prior to an overnight incubation in a $30 \%$ (wt $/ v o l$.) sucrose solution in PBS at $4^{\circ} \mathrm{C}$. The samples were placed on a coverslip, on top of humidified filter paper, in order to avoid dehydration. Different regions were pre- sented to the objective to obtain an overview of the pancreatic organ structures.

Immunohistochemistry Three samples studied by xfOCM were embedded in Tissue-Tek OCT and frozen at $-80^{\circ} \mathrm{C}$. Sections of $8 \mu \mathrm{m}$ thickness were prepared, washed in PBS and blocked with PBS and 10\% FCS for $30 \mathrm{~min}$ at room temperature. Primary antibodies (directed towards: insulin, diluted 1 to 100 [Linco, St Charles, MO, USA]; lectin dolichos biflorus agglutinin (DBA), diluted 1 to 500 [Vector Laboratories, Peterborough, UK]; CD31/ platelet/ endothelial cell adhesion molecule-1, diluted 1 to 50 [BD Biosciences, Allschwil, Switzerland]) were diluted in TBST (Tris-buffered saline and $0.2 \%$ [vol./vol.] Triton X-100) containing $10 \%$ (vol./vol.) FCS, and incubated with the sections overnight at $4^{\circ} \mathrm{C}$. Sections were washed in PBS, incubated for $1 \mathrm{~h}$ with secondary antibodies (Alexa 405, 488 and 555; Invitrogen, Basel, Switzerland) diluted in TBST containing $10 \%$ (vol./vol.) FCS, washed in PBS and mounted. The sections were analysed using a Leica DM 5500 with $\mathrm{a} \times 10$ objective, using excitation wavelengths adapted to each fluorochrome.

Sample preparation from mice injected with streptozotocin C57B6 male mice were controlled for normoglycaemia and then injected once i.p. with either $0.22 \mathrm{mg} / \mathrm{g}$ of freshly prepared streptozotocin (Sigma Chemicals, St Louis, MO, USA) or the corresponding volume $(15 \mu \mathrm{l} / \mathrm{g})$ of the $0.1 \mathrm{mmol} / \mathrm{l}$ citrate buffer ( $\mathrm{pH} 4.5$ ) that was used to dissolve the drug. Thereafter, blood glucose levels of all animals were evaluated every 3 days using a Glucocheck [22].

The animals were killed 4-7 days after the streptozotocin injection and the pancreases were rapidly sampled, weighed and cut into two pieces. One piece was immediately extracted in $5 \mathrm{ml}$ acid ethanol for evaluation of insulin content, as determined by a radioimmunoassay [22]. The other piece was fixed for xfOCM under double-blind conditions.

For xfOCM, the samples were mounted between a coverslip and sample holder, by means of a plastic spacer of $1 \mathrm{~mm}$ thickness. For each sample four regions, each of $1 \times 1.5 \mathrm{~mm}$, were randomly chosen and scanned. The resulting tomogram stacks were then inspected to semiautomatically detect and classify the islets (ESM video 1 shows the overview of all measured samples).

In vivo experiment Adult ICR mice $(n=5)$ (Harlan Laboratories) were anaesthetised with i. p. injection of a solution of $14.5 \mathrm{mg} / \mathrm{kg}$ Rompun (Bayer, Zürich, Switzerland) and $90 \mathrm{mg} / \mathrm{kg}$ kétasol 100 (Dr E. Graeub AG, Bern, Switzerland). The flank skin was shaved and cleaned with Betadine swab. An incision of $0.5-1 \mathrm{~cm}$ was made through the abdominal muscles to gently pull out the duodenum 

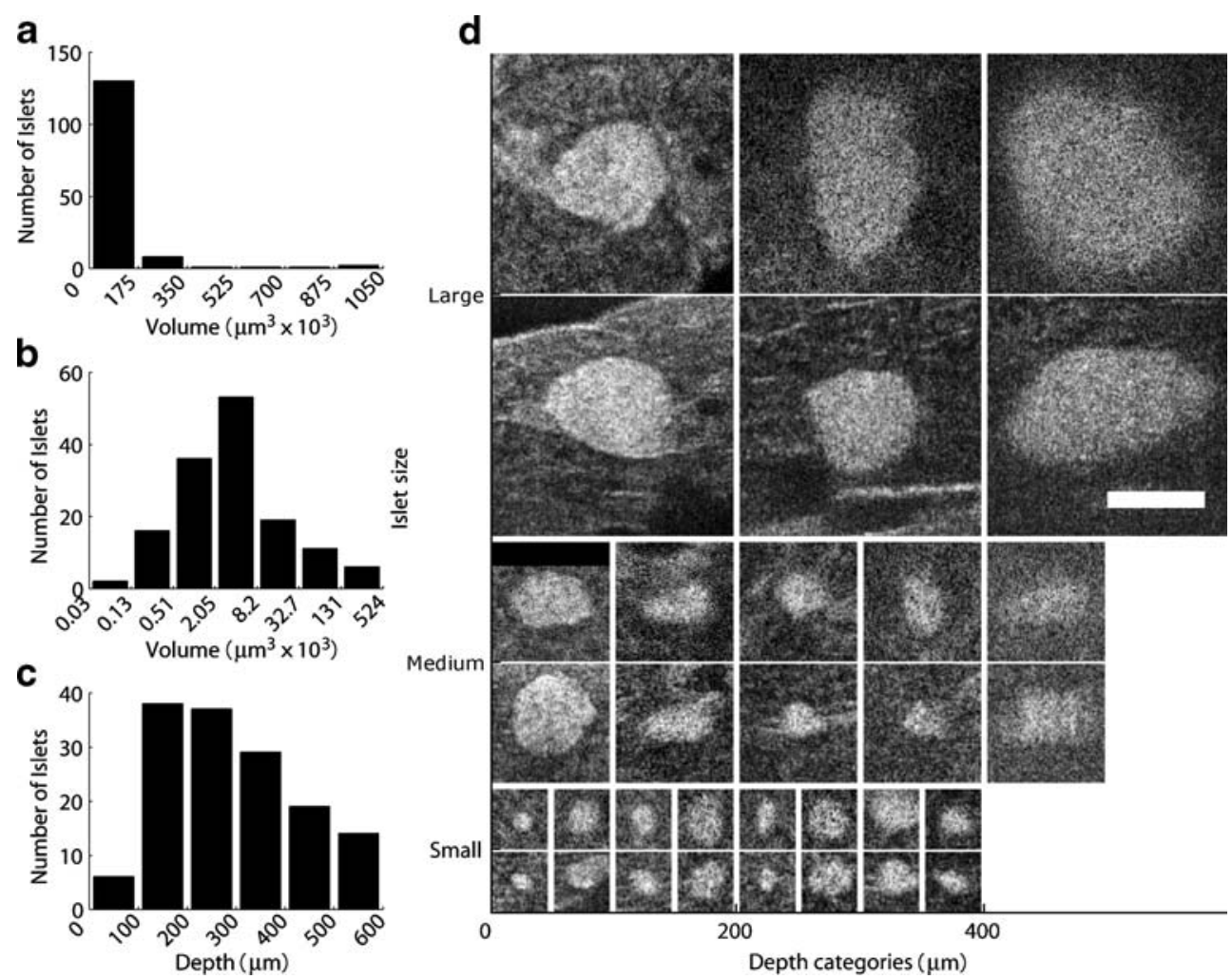

Fig. 3 a Size distribution of the islets in linear scale $\left(n=3,3 \mathrm{~mm}^{3}\right.$ per animal analysed). b The same data as in (a) but on a logarithmic scale, where each size category is four times the volume of the previous one, demonstrating the resolving power of xfOCM. $\mathbf{c}$ Depth distribution of the detected islets. Close to the surface, the pancreas has less islets than in the deeper lying tissue. The reduced number of islets for bigger depths points out the detection limits of the method. d Islets of

encircling the head of the pancreas. The duodenum was stabilised around a pillar during the scanning procedure. The mouse was exposed to a heating lamp and both pancreas and intestine sprayed with $0.9 \% \mathrm{NaCl}$ during the procedure. The measurement was completed and the incision sutured within $30 \mathrm{~min}$ of the start of the operation.

Statistical analysis Results are expressed as means \pm SE. Differences in the mean values of blood glucose and insulin content were tested by analysis of variance using the SPSS program (SPSS, Chicago, IL, USA). Student's $t$ tests with unequal variances were performed to determine the differences in the means of islet contrast and homogeneity.

\section{Results}

Ex vivo imaging of pancreas To investigate the contrast and the structures which can be observed with xfOCM, we excised and fixed the whole pancreases of adult wild-type mice and placed them on microscope slides. The optical setup was different sizes and at different sample depths. Each islet is shown with a en face view (top row) and a lateral scan (bottom row). The depth categories indicate that the islets were found within the first $200 \mu \mathrm{m}$ (large), between $200 \mu \mathrm{m}$ and $400 \mu \mathrm{m}$ (medium), and beyond $400 \mu \mathrm{m}$ (small) in the tissue, respectively. At increasing depth, the lateral resolution is blurred. Scale bar, $100 \mu \mathrm{m}$

mounted on a table in the horizontal direction and the sample was accessed from the side, as indicated in Fig. 2a. The pancreas can be segmented into a tail or splenic part attached to the spleen and a head encircled by the duodenum, connected by a thin neck. Figure $2 \mathrm{~b}$ shows the tomogram of a scan of the neck of a pancreas. Two islets of Langerhans of different sizes are clearly visible as spherical structures with high contrast. The xfOCM signal arises from light scattered by tissue discontinuities. The exocrine tissue has a more granular structure and scatters less light, whereas the ducts and vascular networks appear as dark regions with only their epithelial walls scattering incident light. Adjacent lateral scans were assembled to produce en face images (Fig. 2c), and to generate three-dimensional renderings of the whole volume data, allowing determination of the spatial position and the shape of the islets (Fig. 2d). ESM videos 2 and 3 show, respectively, en face scans at increasing depths and a three-dimensional rendering of the same volume data.

The semi-automatic islet detection helped to exploit this volumetric information and determine the amount of islets and their volume. Four regions of $1.5 \times 1 \mathrm{~mm}$ each were scanned for three different animals. Figure 3 a depicts all 
146 islets distributed into different depth categories. Despite the scattering of the pancreatic tissue, which disturbs the propagation of the incident beam to deeper lying structures and ultimately limits the imaging depth, we could image islets up to more than $500 \mu \mathrm{m}$ in diameter. The histogram of linearly increasing size categories (Fig. 3b) shows that while many small islets were found, only a few larger islets were detected. The same data, but in a logarithmic scale, are displayed in Fig. 3c. Islets down to a size of only about $15 \mu \mathrm{m}$ in diameter $\left(\sim 2000 \mu \mathrm{m}^{3}\right)$, corresponding to an aggregate of only a few cells, were reliably detected.

Figure $3 \mathrm{~d}$ presents an assembly of tomograms from islets of different sizes and depths, illustrating the resolving power of xfOCM.

\section{Validation of xfOCM tomograms by immunohistochemistry} To ascertain that the spherical structures were indeed islets, we sectioned a subset of the samples. Figure 4 shows alignments between immunostained sections and virtual sections through an xfOCM stack. Cryosections were immunolabelled to identify beta cells (using anti-insulin antibody), ducts (using anti-DBA-lectin antibody) and
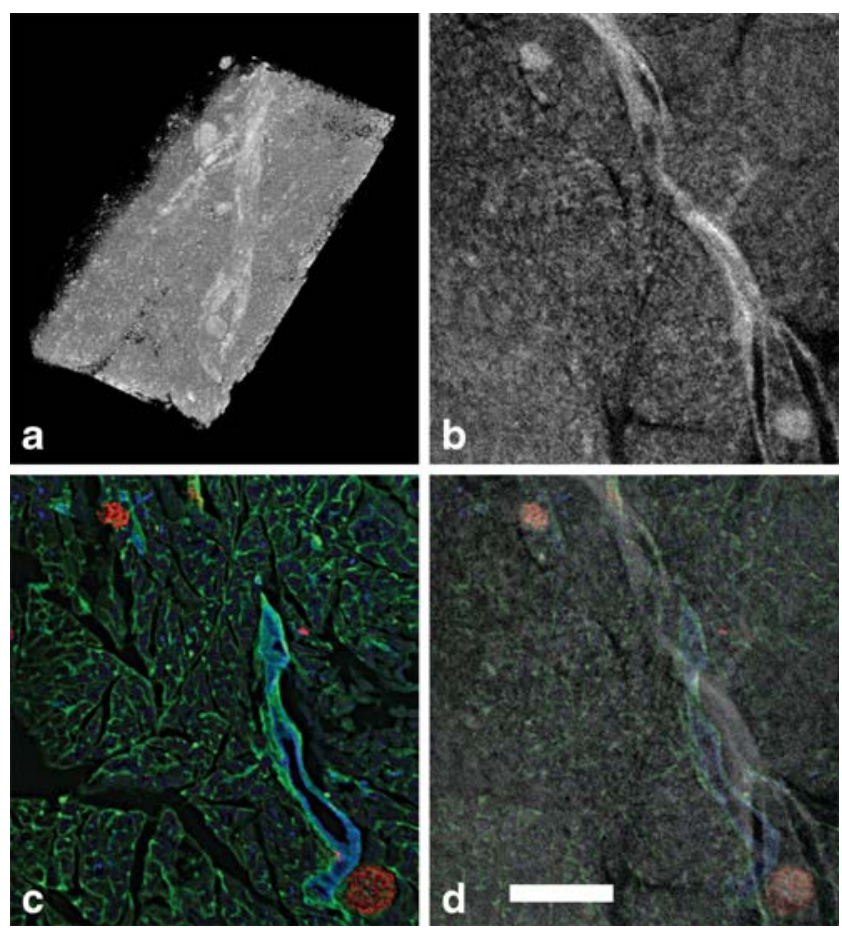

Fig. 4 a Three-dimensionally rendered volume $(0.86 \times 1.7 \times 0.78 \mathrm{~mm})$ of the pancreas head. b Virtual section of the tomographic data. c Cryosection of the same area, immunohistochemically labelled for insulin (red), platelet/endothelial cell adhesion molecule-1 (PECAM1) (green) and DBA lectin (blue). d Overlay of cryosection with xfOCM data identifying the islets and vessel structures. Identical scale bar of $250 \mu \mathrm{m}$ for $\mathbf{b}, \mathbf{c}$ and $\mathbf{d}$ blood vessels (using anti-platelet/endothelial cell adhesion molecule-1 [PECAM] antibody). Overlays showed good correspondence between the cryosections and the virtual sections from the xfOCM data (Fig. 4). The few unmatched differences result from the distortions that tissues undergo during fixation and cryosectioning, and were compensated by a slight scaling of the xfOCM data. This demonstrates the advantage of imaging the tissue structure in fresh, unfixed tissue, where the native tissue morphology is revealed. This benefit has also been stressed in the context of other in vivo imaging studies [23].

Imaging of mice injected with streptozotocin To determine whether xfOCM could detect changes in beta cell mass, we injected a group of mice $(n=7)$ with streptozotocin, a drug which selectively kills insulin-producing cells [24]. A control group $(n=6)$ was injected with the citrate buffer that served as the vehicle for the drug. Four to 7 days after injection all control mice showed normal levels of blood glucose $(8.6 \pm 1 \mathrm{mmol} / \mathrm{l}, n=6)$ and total insulin content $(58.8 \pm 15.4 \mu \mathrm{g}$ per pancreas, $n=6)$ (Fig. $5 \mathrm{a}$, f). In contrast, the mice that had received streptozotocin showed markedly increased $(p<0.002)$ blood glucose levels $(25.47 \pm$ $3.27 \mathrm{mmol} / \mathrm{l}, n=7)$ and a significantly lower $(p<0.001)$ total insulin content $(25.9 \pm 15.6 \mu \mathrm{g}$ per pancreas, $n=7)$ (Fig. 5a, f).

The xfOCM analysis of the samples, carried out under double-blind conditions, showed that some of the pancreases contained numerous islets whereas others contained significantly fewer islets $(p<0.001$; Fig. $5 b)$. Opening of the codes revealed that all of the pancreases of the former type were from control mice with normal glucose homeostasis, whereas all the samples of the latter type suffered from hyperglycaemia resulting from streptozotocin injection.

The few islets remaining after streptozotocin treatment were of various sizes without privileged volume category. (Fig. 5g). Mean values for contrast and islet homogeneity were significantly different from the corresponding values for the control islets $(p<0.001)$ (Fig. 5c, h) when only islets larger than $1,000 \mu^{3}$ were included. Figure $5 \mathrm{~d}, \mathrm{e}, \mathrm{i}, \mathrm{j}$ shows two selected islets of approximately the same size and depth in the tissue, indicating the difference in homogeneity of control and treated islets. These experiments demonstrate that xfOCM can detect beta cell loss and alterations in streptozotocin-treated animals.

Imaging of pancreas in vivo Finally, we scanned the head of the pancreas in vivo after laparotomy of anaesthetised mice. To increase the scanning speed, small regions of $256 \times 256$ pixels were scanned with low sampling in less than $5 \mathrm{~s}$. The few remaining movement artefacts were corrected with an image registration algorithm [25]. After imaging, the incision was sutured allowing the mouse to recover. 
An overview of samples measured in vivo is shown in Fig. 6. With the three-dimensional data it is possible to derive the shape of the islets and their position relative to ducts, blood vessels and pancreatic lobes (Fig. 6d and ESM videos 4 and 5). The semi-automatically detected islets were analysed and revealed a stronger absorption of tissue in the living organism, limiting the imaging depth to about $300 \mu \mathrm{m}$, as indicated in Fig. 6f.

\section{Discussion}

In this study, we first characterised the imaging capacity of xfOCM by measuring fixed and excised pancreatic tissue. Comparison with histology confirmed that xfOCM can detect islets of all sizes and their association with ducts and blood vessels. Next, we found that xfOCM can discriminate between the islets of animals that had normal insulin content from the mice suffering from hyperglycaemia and decreased insulin content as a result of the beta cell death induced by streptozotocin. The data show that XfOCM can detect changes in the number of islets and in the contrast and homogeneity of the few residual islets that survive the cytotoxic effect of the drug. Measurements made from living mice showed that xfOCM can visualise pancreatic lobules, ducts, blood vessel networks and islets of Langerhans in vivo.
OCT is a well established clinical technique in ophthalmology for retinal imaging and is gaining importance for minimally invasive imaging of other biological tissues. OCT has been reported in an earlier work to image fixed pancreatic tissue and detect islets [26]. However, only the high, near isotropic, resolution of xfOCM in combination with the in-parallel depth extraction giving high-speed imaging allows in vivo imaging and generates tomograms exhibiting high contrast. This contrast is generated by an intrinsic sample property without any exogenous labelling. Hence, individual islets were visualised in the living organism as well as in fixed tissue. The exact nature of the strong scattering of the islets remains unclear. Whether it is the whole structure of the beta cell that strongly scatters the light, as indicated by the streptozotocin experiments, or only the insulin-containing vesicles within the beta cells has to be shown by further experiments.

A recently published work [27] shows a procedure for imaging the pancreas in vivo with two-photon microscopy and involves gluing the pancreas between two glass plates. In contrast, once the pancreas is exposed, xfOCM works contact free without immersion or coverslips and requires only slight stabilisation of the sample to suppress the breathing motion of several millimetres. Repeated measurements should be feasible, providing that the same area of the pancreas can be identified sequentially. For such longitudinal studies, the access to the pancreas could be simplified by
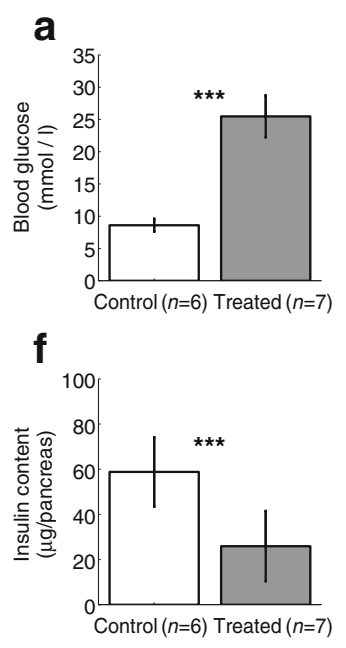

Fig. 5 Exposure of C57B6 mice to streptozotocin (grey bars) resulted in a marked increase in blood glucose levels (a) and in a parallel decrease in the insulin content of the pancreas (f) compared with the control group (white bars). The selective beta cell death induced by the drug also decreased the number of islets found in the analysed regions of interest (b, screened tissue volume of $2.5 \mathrm{~mm}^{2}$ per animal). g The logarithmic distribution of the islet size exhibits a characteristic pattern for the control islets that is lost in the case of the remaining islets of treated animals. The few remaining islets (only islets
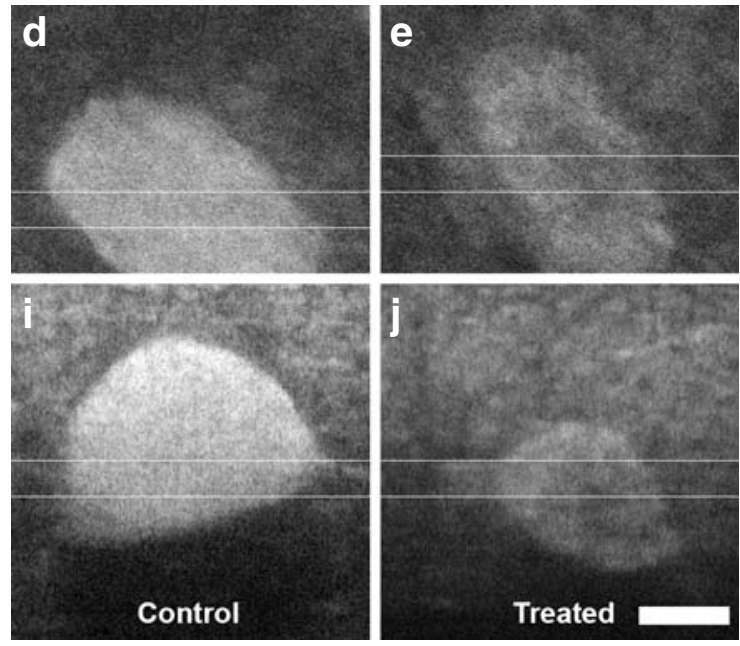

$>1000 \mu^{3}$ taken into account) likewise show a reduced contrast (c) and homogeneity (h). (a, b, c, f and $\mathbf{h}$ show means $\pm \mathrm{SE}, * * * p<$ 0.001). Results from control mice are shown by the white bars and results for the treated mice are shown in grey. An islet surviving in a diabetic mouse $(\mathbf{e}, \mathbf{j})$ shows a significant reduction in contrast, compared to a control islet $(\mathbf{d}, \mathbf{i})$. The columns show en-face views $(\mathbf{d}, \mathbf{e})$ and lateral scans (i, j), averaged over the 20 slides indicated by the white lines. The lateral scans are adjusted in their axial position to show identical sample depths. The scale bar shows $100 \mu \mathrm{m}$ and applies to all tomograms 

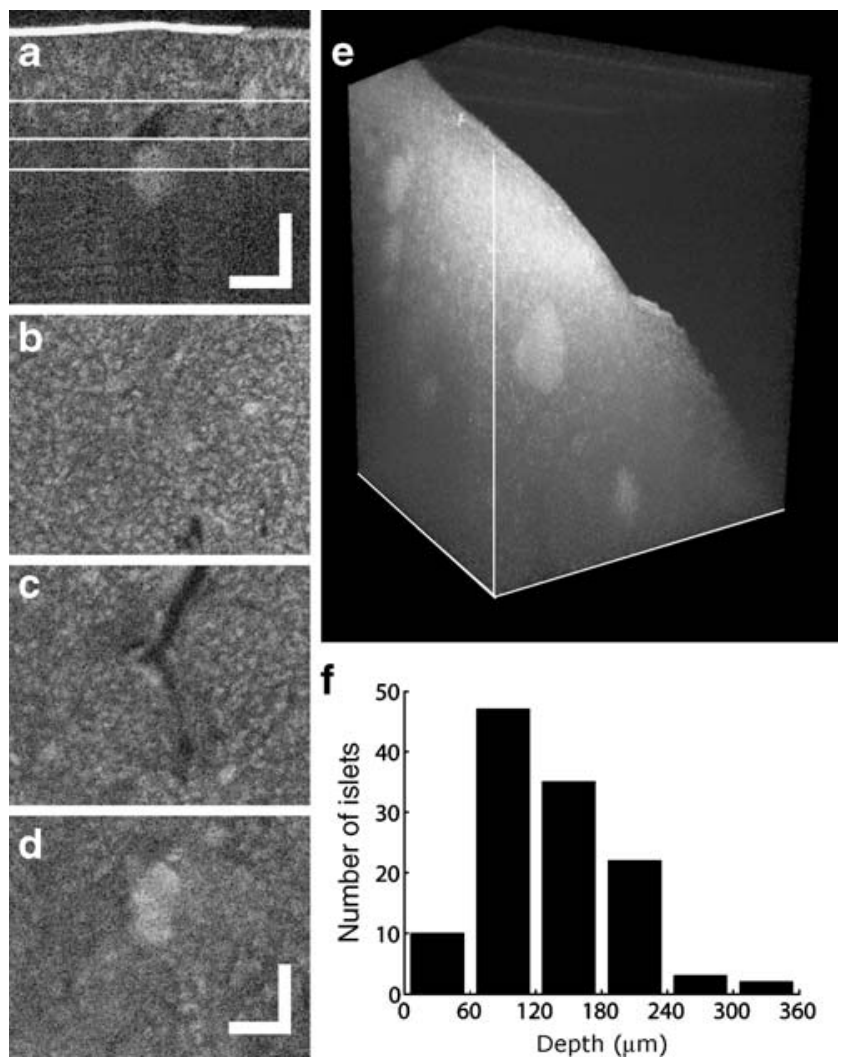

Fig. 6 In vivo imaging of islets. a Side view and (b, c, d) corresponding en-face views (indicated by the white lines in the side view, median value over five slices) at different depths, showing islets and a duct- or vessel-like structure. The scale bars show $100 \mu \mathrm{m}$ and apply to all views. e Three-dimensional rendering of a similar in vivo tomogram showing several islets at various depths, contained in a lobe of a pancreas. The volume measures $1 \mathrm{~mm} \times 1 \mathrm{~mm}$ in the lateral directions and $500 \mu \mathrm{m}$ in the vertical axis. $\mathbf{f}$ The depth distribution of the islets detected in vivo (six animals, 123 islets)

employing an endoscope or a glass window in the abdominal wall, making our method minimally invasive.

With the three-dimensional volumetric data at hand, quantitative measurements of islet volume, shape and signal strength were possible in a semi-automatic way. This analysis enabled the detection of subtle islet alterations, and provided islet size distributions. The well-known exponential distribution of islet sizes [e.g. 7] was not matched here, because only a limited amount of the whole islet population was assessed. However, judging from the diameter of the smallest islets detected $(15 \mu \mathrm{m})$, the resolution of $\mathrm{xfOCM}$ is one order of magnitude higher than that of OPT [7].

Furthermore, the quantitative analysis estimated the imaging depth, which was more limited in living than in fixed samples. The depths achieved are comparable with those reported for two-photon microscopy. A trade-off between a light source in a longer wavelength region and the resulting reduced axial resolution could further optimise imaging performance. Even then, it is unlikely that xfOCM could measure the whole volume of the pancreas. Still, the surface volume of the pancreas is screened with unprecedented accuracy, offering new insight into this organ. Essentially, with xfOCM the islets are studied in their natural environment, and disease evolution and islet destiny can be evaluated. This is in contrast to the anterior eye chamber imaging platform [12] that is limited to the study of the engraftment process and graft function in an immunologically privileged engraftment site. Although lacking molecular specificity, xfOCM is potentially able to measure islets in any site accessible to the microscope, provided the islets contrast with the surrounding tissue. Also, xfOCM could be combined with two-photon microscopy to provide the scattering and fluorescence signal in parallel.

The newest generation of line cameras further increase the imaging speed of $x f O C M$ and will enable the monitoring of islets in real time to study the response to stimuli or drug administration. This increased speed will also enhance the capacity of OCT to perform Doppler flow measurements, a technique we and others have previously used in the eye [28, 29]. This principle could be adapted to reveal the pancreatic blood flow and provide additional functional variables. The sensitivity of xfOCM should prove useful to monitor changes in beta cell number-for example, during islet regeneration [30] or upon islet transplantation to visualise the islets and their vascularisation in the recipient. Analysing dispersion and spectral absorption characteristics in the sample could add further functional contrast such as variations in metabolite concentrations [31,32]. Eventually, xfOCM could help design new PET and MRI probes that selectively label the pancreatic beta cells and that are necessary for clinical imaging of pancreatic islets by these techniques. Adding a contrast agent for xfOCM to prospective probes would enable the monitoring of their dynamics and specificity on a microscopic scale.

Acknowledgements This research was funded by the Swiss National Science Foundation (SNF) and an EU grant (SNF grants 205321-10974, 205320-121022/1, EU FP7-222980). J. Goulley is funded by the Beta Cell Biology Consortium (Grant NIDDK U19 DK072495-02). P. Meda is further supported by grants from the SNF (310000-122430), the EU (IMIDIA, C2008-T7) and the juvenile diabetes research foundation (JDRF 1-2207-158). R. A. Leitgeb is also funded by an EU grant (FP7201880). We would like to thank: W. Pralong (Life Sciences, Ecole Polytechnique Fédérale de Lausanne, Switzerland) for valuable discussions; G. Ferrand (Life Sciences, Ecole Polytechnique Fédérale de Lausanne, Switzerland) for her veterinary expertise; A. Lopez (Laboratoire d'Optique Biomédicale, Ecole Polytechnique Fédérale de Lausanne, Switzerland) for his work on the figure preparation; and E. MartinWilliams (Laboratoire d'Optique Biomédicale, Ecole Polytechnique Fédérale de Lausanne, Switzerland) for critical reviewing.

Duality of interest The authors declare that there is no duality of interest associated with this manuscript. 


\section{References}

1. Souza F, Freeby M, Hultman K et al (2006) Current progress in non-invasive imaging of beta cell mass of the endocrine pancreas. Curr Med Chem 13:2761-2773

2. Evgenov NV, Medarova Z, Dai GP, Bonner-Weir S, Moore A (2006) In vivo imaging of islet transplantation. Nat Med 12:144-148

3. Tai JH, Foster P, Rosales A et al (2006) Imaging islets labeled with magnetic nanoparticles at 1.5 Tesla. Diabetes 55:2931-2938

4. Kim SJ, Doudet DJ, Studenov AR et al (2006) Quantitative micro positron emission tomography (PET) imaging for the in vivo determination of pancreatic islet graft survival. Nat Med 12:1423-1428

5. Holmberg D, Ahlgren U (2008) Imaging the pancreas: from ex vivo to non-invasive technology. Diabetologia 51:2148-2154

6. Sharpe J, Ahlgren U, Perry P et al (2002) Optical projection tomography as a tool for 3D microscopy and gene expression studies. Science 296:541-545

7. Alanentalo T, Asayesh A, Morrison H et al (2007) Tomographic molecular imaging and $3 \mathrm{D}$ quantification within adult mouse organs. Nat Methods 4:31-33

8. Dodt HU, Leischner U, Schierloh A et al (2007) Ultramicroscopy: three-dimensional visualisation of neuronal networks in the whole mouse brain. Nat Methods 4:331-336

9. Hermann M, Pirkebner D, Draxl A, Margreiter R, Hengster P (2005) 'Real-time' assessment of human islet preparations with confocal live cell imaging. Transplant Proc 37:3409-3411

10. Boffa DJ, Waka J, Thomas D et al (2005) Measurement of apoptosis of intact human islets by confocal optical sectioning and stereologic analysis of YO-PRO-1-stained islets. Transplantation 79:842-845

11. Takahashi N, Nemoto T, Kimura R et al (2002) Two-photon excitation imaging of pancreatic islets with various fluorescent probes. Diabetes 51:S25-S28

12. Speier S (2008) Noninvasive in vivo imaging of pancreatic islet cell biology. Nat Med 14:574-578

13. Hitzenberger CK (1991) Optical Measurement of the Axial Eye Length by Laser Doppler Interferometry. Invest Ophthalmol Vis Sci 32:616-624

14. Huang D, Swanson EA, Lin CP et al (1991) Optical coherence tomography. Science 254:1178-1181

15. Leitgeb RA, Villiger M, Bachmann AH, Steinmann L, Lasser T (2006) Extended focus depth for Fourier domain optical coherence microscopy. Opt Lett 31:2450-2452

16. Fercher AF, Hitzenberger CK, Kamp G, Elzaiat SY (1995) Measurement of intraocular distances by backscattering spectral interferometry. Opt Commun 117:43-48

17. Leitgeb R, Hitzenberger CK, Fercher AF (2003) Performance of Fourier domain vs time domain optical coherence tomography. Opt Express 11:889-894
18. Yun SH, Tearney GJ, Vakoc BJ et al (2006) Comprehensive volumetric optical microscopy in vivo. Nat Med 12:1429-1433

19. Jenkins MW, Adler DC, Gargesha M et al (2007) Ultrahigh-speed optical coherence tomography imaging and visualisation of the embryonic avian heart using a buffered Fourier Domain Mode Locked laser. Opt Express 15:6251-6267

20. Izatt JA, Hee MR, Owen GM, Swanson EA, Fujimoto JG (1994) Optical coherence microscopy in scattering media. Opt Lett 19:590-592

21. Huang SW, Aguirre AD, Huber RA, Adler DC, Fujimoto JG (2007) Swept source optical coherence microscopy using a Fourier domain mode-locked laser. Opt Express 15:6210-6217

22. Charollais A, Gjinovci A, Huarte J et al (2000) Junctional communication of pancreatic beta cells contributes to the control of insulin secretion and glucose tolerance. J Clin Invest 106:235243

23. Vincent P, Maskos U, Charvet I et al (2006) Live imaging of neural structure and function by fibred fluorescence microscopy. EMBO Rep 7:1154-1161

24. Lenzen S (2008) The mechanisms of alloxan- and streptozotocininduced diabetes. Diabetologia 51:216-226

25. Thevenaz P, Ruttimann UE, Unser M (1998) A pyramid approach to subpixel registration based on intensity. IEEE Trans Image Process 7:27-41

26. Tearney GJ, Brezinski ME, Southern JF, Bouma BE, Boppart SA, Fujimoto JG (1998) Optical biopsy in human pancreatobiliary tissue using optical coherence tomography. Dig Dis Sci 43:11931199

27. Martinic MM, von Herrath MG (2008) Real-time imaging of the pancreas during development of diabetes. Immunol Rev 221:200213

28. Leitgeb RA, Schmetterer L, Drexler W, Fercher AF, Zawadzki RJ, Bajraszewski T (2003) Real-time assessment of retinal blood flow with ultrafast acquisition by color Doppler Fourier domain optical coherence tomography. Opt Express 11:3116-3121

29. Bachmann AH, Villiger ML, Blatter C, Lasser T, Leitgeb RA (2007) Resonant Doppler flow imaging and optical vivisection of retinal blood vessels. Opt Express 15:408-422

30. Moogk D, Hanley S, Ramunas J et al (2007) Design and analysis of a long-term live-cell imaging chamber for tracking cellular dynamics within cultured human islets of Langerhans. Biotechnol Bioeng 97:1138-1147

31. Esenaliev RO, Larin KV, Larina IV, Motamedi M (2001) Noninvasive monitoring of glucose concentration with optical coherence tomography. Opt Lett 26:992-994

32. Leitgeb R, Wojtkowski M, Kowalczyk A, Hitzenberger CK, Sticker M, Fercher AF (2000) Spectral measurement of absorption by spectroscopic frequency-domain optical coherence tomography. Opt Lett 25:820-822 